Construire le voisin. Pratiques européennes

\title{
Définir le voisin. La genèse de la Politique européenne de voisinage
}

Alternative narratives of the European neighbourhood policy

Julien Jeandesboz

\section{(2) OpenEdition \\ Journals}

Édition électronique

URL : http://journals.openedition.org/conflits/2441

DOI : $10.4000 /$ conflits. 2441

ISSN : $1777-5345$

Éditeur :

CCLS - Centre d'études sur les conflits lilberté et sécurité, L'Harmattan

Édition imprimée

Date de publication : 17 septembre 2007

Pagination : 11-29

ISBN : 978-2-296-03458-7

ISSN : 1157-996X

Référence électronique

Julien Jeandesboz, «Définir le voisin. La genèse de la Politique européenne de voisinage », Cultures \& Conflits [En ligne], 66 | été 2007, mis en ligne le 05 juillet 2007, consulté le 30 mars 2021. URL : http:// journals.openedition.org/conflits/2441; DOI : https://doi.org/10.4000/conflits.2441 


\section{Définir le voisin. La genèse de la Politique européenne de voisinage ${ }_{1}$}

\section{Julien JEANDESBOZ}

Julien Jeandesboz est doctorant en science politique et relations internationales à l'Institut d'études politiques de Paris, et chercheur associé an Centre d'études sur les conflits.

$\mathrm{D}$

ans son rapport de 2003, adressé entre autres à «ceux qui vont concevoir et construire la politique de voisinage de l'Union européenne ", le Groupe des sages rassemblé à l'initiative de Romano Prodi pour réfléchir sur la teneur d'un dialogue entre les peuples et les cultures en Méditerranée souligne :

« Le retour du politique parât nécessaire. Le 11 septembre 2001 a rappelé cette évidence de façon spectaculaire [...]. Ce retour du politique constitue aussi une réponse à d'autres manifestations meurtrières du radicalisme en Méditerranée : les guerres des Balkans, le conflit israélo-palestinien, la montée des terrorismes et la lente réponse sécuritaire à la peur engendrée par ces situations. Dans un monde auquel la globalisation promet un avenir régi par l'économie, la Méditerranée propose quant à elle l'aspiration des peuples à l'expression démocratique et à la défense des droits, c'est-à-dire la primanté du "bien commun" régi par le politique 2 ".

1. Ce texte est la version traduite et remaniée d'une intervention intitulée Alternative narratives of the European neighbourhood policy donnée à la première école doctorale CHALLENGE «Perspectives on the European neighbourhood policy » au Centre for European Policy Studies de Bruxelles les 21 et 22 avril 2006. L'auteur souhaite remercier les participants à cette conférence, et tout particulièrement Sandra Lavenex, pour leurs commentaires et propositions, ainsi que Thierry Balzacq, en sa qualité d'organisateur de la conférence et de directeur de numéro. Enfin, un dernier remerciement à Stephan Davidshofer pour ses relectures et conseils, ainsi qu'à deux lecteurs anonymes pour leurs suggestions.

2 . Groupe des sages sur le dialogue interculturel, Le Dialogue entre les peuples et les cultures dans l'espace euro-méditerranéen, Bruxelles, octobre 2003, disponible en ligne : http://www.libertysecurity.org/IMG/pdf/rapport_complet_fr.pdf, p. 16, soulignement d'origine. 
Le texte se distingue à la fois par la situation de ses rédacteurs, qui occupent des positions sociales éloignées des arènes communautaires, et par son appel à un traitement politique des questions relevant du vivre-ensemble en Méditerranée, et notamment des insécurités, qui contraste avec la focale technique, axée sur la résolution de problèmes, généralement adoptée par les documents officiels ayant trait à la politique européenne de voisinage (PEV).

Ce décalage est à mettre en relation avec le fait que cette contribution ait été presque entièrement ignorée par ceux auxquels elle était pourtant destinée. La marginalisation d'une prise de position ouvertement alternative aux orientations des relations entre l'UE et ses partenaires méditerranéens (et au-delà, avec ses autres « voisins ») illustre bien l'importance de s'attacher aux conditions qui ont rendu possibles le lancement et l'installation de la politique de voisinage dans les arènes européennes, en la considérant non pas comme une réponse à une situation objectivement donnée, mais plutôt en tant que processus de construction réciproque de solutions et de problèmes. Il ne s'agit donc pas ici de réfléchir à la PEV en termes de «défis ${ }^{3}$ » à relever, ni en termes d' «instruments » ou de "valeur ajoutée 4 ». Nous apportons en revanche un regard sociologique, en proposant une lecture socio-historique, inscrite dans une logique processuelle et relationnelle, de la genèse de la PEV. Nous nous concentrons à la fois sur la formulation du voisinage 5 , c'est-à-dire sur les pratiques discursives qui confèrent du sens à la notion de voisinage, et sur les usages qui sont faits de ces discours dans les jeux sociaux qui s'organisent autour de la mise en place de la PEV. Pour ce faire, nous considérons l'élaboration de la politique de voisinage depuis la multiplication des propositions pour une initiative de ce type dans les premiers mois de l'année 2002, jusqu'à son installation sous sa forme actuelle au travers de l'adoption des premiers plans d'action en décembre 2004.

\section{Formuler le voisinage : discours autorisés sur la PEV}

La PEV est formellement lancée en mars 2003 par une communication de la Commission intitulée $L^{\prime}$ Europe élargie - Voisinage ${ }^{6}$. Il faut néanmoins souli-

3. Comelli M., "The challenges of the European Neighbourhood Policy», The International Spectator, vol. XXXIX, n³, 2004, pp. 97-110; Koopmann M., « La politique de voisinage de l'Union européenne élargie : ambitions et défis ", Allemagne d'aujourd'hui, n¹71, 2005, pp. 57 69 ; Tocci N., «Does the ENP respond to the EU's post-enlargement challenge?», The International Spectator, vol. XL, $\mathrm{n}^{\circ} 1,2005$, pp. 21-32.

4 . Balfour R., Rotta A., «Beyond enlargement. the European Neighbourhood Policy and its tools ", The International Spectator, vol. XL, ${ }^{\circ}$ 1, 2005, pp. 7-20; Kelley J., « New wine in old wineskins: promoting political reforms through the New European Neighbourhood Policy ", Journal of Common Market Studies, vol. 44, $\mathrm{n}^{\circ}$ 1, 2006, pp. 29-55.

5. A l'exemple de ce que propose Alexandra Goujon dans son analyse de la mise en place de la PEV, qui s'inspire en revanche de la notion de narrations de politiques publiques. Voir Goujon A., «L'Europe élargie en quête d'identité : légitimation et politisation de la politique européenne de voisinage », Politique européenne, $\mathrm{n}^{\circ} 15,2005$, pp. 137-163.

6. Commission européenne, L'Europe élargie - Voisinage : un nouveau cadre de relations avec nos voisins de l'Est et du Sud, Bruxelles, COM(2003) 104 final, 11 mars 2003, disponible en ligne : http ://europa.eu.int/comm/energy_transport/euromed_conf3/doc/com_2003_0262_en.pdf 
gner que ce document s'inscrit dans la continuité d'interventions s'étant multipliées à partir de 20027 . Ces interventions divergent sur les pays à prendre en compte, sur la lecture qu'elles proposent des relations entre ceux-ci et l'UE, et sur les orientations à donner à une éventuelle initiative. Elles partagent toutes, en revanche, la même technique de légitimation, à savoir la nécessité d'une évolution dans les relations entre l'UE et ses contiguïtés géographiques à la lumière de l'élargissement prévu de l'Union à dix nouveaux membres en 2004. Elles convergent également sur l'inscription de la question du voisinage au sein des arènes communautaires. Elles reflètent donc, de fait, un usage similaire de l'intériorité et de l'extériorité quant au statut des voisins vis-à-vis du processus d'intégration communautaire, en les positionnant dans un entre-deux au caractère ambigu, dont la qualification devient alors l'enjeu central de la mise en place de la PEV. Elles dessinent à ce titre un espace de prises de position complexe, au sein duquel nous suggérons néanmoins qu'il est possible de distinguer une ligne d'opposition fondamentale, autour des questions et pratiques de sécurité.

Les liens unissant la PEV à l'agenda comme aux pratiques de sécurité au niveau européen ont été soulignés ailleurs ${ }^{8}$. L'enjeu qui nous préoccupe ici, outre le fait de souligner certains éléments restés peu traités, est de mettre en relation des prises de positions, au sein desquelles les usages de la sécurité constituent un clivage majeur, avec les positions depuis lesquelles elles ont été formulées. Deux interventions au cours de l'année 2002 permettent de résumer ces prises de position. Dans une lettre adressée en janvier 2002 à son homologue espagnol Josep Piqué, dont le gouvernement occupait à l'époque la présidence tournante de l'Union, le ministre des Affaires étrangères britannique Jack Straw souligne :

\begin{abstract}
«Sous trois ans, l'Ukraine et la Biélorussie seront à la frontière de l'UE - avec tous les problèmes attenants de criminalité transfrontalière, de trafic et d'immigration illégale. La Moldavie ne sera un voisin de l'Union que plus tard [...], mais elle est déjà confrontée à une pauvreté écrasante, d'importants problèmes sociaux et une émigration massive ${ }^{9}$ ».
\end{abstract}

7. Pour des questions de longueur, cet article ne traite pas des développements antérieurs à janvier 2002. Il est cependant clair que les thématiques qui structurent le traitement de la question du voisinage participent d'un processus beaucoup plus long, qui porte sur la définition des limites géographiques et institutionnelles du processus d'intégration communautaire. A ce titre, il conviendrait de revenir plus longuement sur les débats liés à l'élargissement de l'UE proprement dit, mais également sur les différents aménagements, « internes » (Europe «à la carte », en « cercles concentriques » des « coopérations renforcées » ou des « groupes pionniers ») et « externes » (les relations entre l'UE et les autres espaces institutionnels dits européens, comme le Conseil de l'Europe ou l'Espace économique européen), proposés au cours des années 1990.

8 . Voir, dans une perspective relativement classique : Lynch D., «The security dimension of the European Neighbourhood Policy », The International Spectator, vol. XL, n`1, 2005, pp. 31-43; Aliboni R., "The geopolitical implications of the European Neighbourhood Policy », European Foreign Affairs Review, vol. 10, $\mathrm{n}^{\circ} 1$, pp. 1-16. Pour une lecture plus critique, voir l'article de R. Zaiotti dans ce numéro.

9. Foreign and Commonwealth Office, Letter from Jack Straw to Josep Piqué, Londres, 28 janvier 2002. Notre traduction. 
La lettre propose "une sorte de "statut spécial de voisin" ancré dans un attachement aux principes de la démocratie et de l'économie de marché » qui offrirait "une libéralisation des échanges commerciaux, une relation plus étroite sur la justice et les affaires intérieures, et un dialogne politique privilégié qui inclurait une coopération plus approfondie en matière de PESC ${ }^{10}$. Les questions de frontières et de migration, et leur gestion dans une logique de sécurité exprimée en termes de contrôle, occupent dans cette première orientation une place prépondérante ${ }^{11}$.

Cette première prise de position contraste fortement avec une autre intervention, cette fois-ci de Romano Prodi, président, à l'époque, de la Commission européenne. Dans une allocution à Louvain-la-Neuve en novembre 2002, sur les perspectives du partenariat euro-méditerranéen, Prodi établit la distinction suivante :

« Nous avons le choix entre deux possibilités très différentes l'une de l'autre [...]. La première consiste à considérer la Méditerranée principalement en termes de sécurité. Il s'agit alors de la frontière méridionale de l'Union, sur laquelle il faut se placer pour gérer les flux migratoires, combattre l'éventuelle diffusion du terrorisme international et encourager une politique de développement fortement dépendante de la coopération contre les différentes activités illégales. C'est l'approche du flanc sud longtemps chère à l'OTAN [...]. La seconde consiste à considérer la Méditerranée principalement comme un nouveau domaine de coopération, où il convient d'établir des relations spéciales dans le cadre d'une politique plus large de voisinage, qui impliquera toutes les régions situées en bordure de l'Union, du Maghreb à la Russie ${ }^{12}$ ».

Cette seconde perspective organise une tension entre deux lectures des interactions impliquant le processus d'intégration européen d'une part, et les espaces politiques avec lesquels il entre en contact d'autre part. Si la première se rappro-

10. Ibid. Notre traduction.

11. Cette insistance sur les questions dites de Justice et Affaires intérieures s'inscrit par ailleurs dans la ligne des évolutions observables pour ce qui concerne les relations entre l'UE et les pays mentionnés dans la lettre. Ainsi, le Conseil de l'UE avait déjà adopté, le 10 décembre 2001, un plan d'action en matière de justice et affaires intérieures qui s'ajoutait à l'accord de partenariat et de coopération ratifié en 1998 entre l'UE et l'Ukraine. Ce texte, cependant, ne sera finalement adopté qu'en 2003, et publié au journal officiel de mars 2003. Voir : Conseil de l'Union européenne, Plan d'action de l'Union européenne dans le domaine de la Justice et des Affaires intérieures en Ukraine, Bruxelles, 2003/C 77/01, 29 mars 2003, disponible en ligne :

http://eur-lex.europa.eu/LexUriServ/site/fr/oj/2003/c_077/c_07720030329fr00010005.pdf

12 . Prodi R., L'Europe et la Méditerranée : venons-en aux faits, Louvain-la-Neuve, 26 novembre 2002, Speech 02/589, disponible en ligne :

http://europa.eu/rapid/pressReleasesAction.do?reference=SPEECH/02/589\&format $=\mathrm{PDF} \&$ aged $=1 \&$ language $=\mathrm{FR} \&$ guiLanguage $=\mathrm{en}, \mathrm{p} .3$. Soulignement d'origine. 
che des positions exprimées dans la lettre Straw (focalisation sur la gestion des frontières et le contrôle des flux migratoires, association du développement et de la sécurité), la seconde, a contrario, se caractérise avant tout par le rejet d'un lien direct entre une éventuelle politique de voisinage et un agenda sécuritaire.

Les prises de position relatives à cette seconde lecture vont occuper le devant de la scène dans les derniers mois de l'année 2002. Le discours de Romano Prodi à Louvain-la-Neuve fait partie d'une série d'interventions du président de la Commission sur le thème des relations entre l'UE et ses contiguités ${ }^{13}$, qui s'échelonnent courant 2002. Cette série s'achève avec une allocution devant la conférence mondiale de la European Community Studies Association (ECSA) des 5-6 décembre $2002{ }^{14}$, qui développe plus avant l'idée d'une "politique plus large de voisinage». Cette politique est avant tout constituée comme un enjeu interne majeur, et notamment comme une orientation alternative à la pratique de l'élargissement supposée menacer l'intégrité du projet européen: "Nous ne pouvons toutefois continuer éternellement [...] avec le seul instrument de l'élargissement. Nous ne pouvons pas diluer le projet politique européen et transformer l'Union européenne en une simple zone de libre-échange à l'échelle du continent 15 ». Il s'agit alors, dans cette logique, de déterminer «le projet politique le plus susceptible d'étendre la zone de stabilité [de l'UE] sans élargissement immédiat de l'Union 16 ", pour servir un triple objectif de stabilité, de prospérité et de sécurité.

Ce projet est formulé en termes de rapprochement, autour de la notion de partage : partage, d'une part, de «valeurs communes », telles que la démocratie, le respect de l'Etat de droit, les libertés civiles et les droits de l'Homme; partage, d'autre part, des «quatre libertés» (de circulation des biens, des capitaux, des services, et plus important encore, des personnes) qui constituent le socle du marché commun européen. Il s'agit ici, sur la base d'un agenda de réformes politiques (inspiré par les «valeurs communes » déjà évoquées) et socio-économiques (passant notamment par l'adoption dans la législation nationale des voisins de certaines parties de l'acquis communautaire), d'établir un « cercle de pays amis », dans un cadre qui serait «davantage qu'un partenariat, mais moins

13. Voir pour référence : Prodi R., L'Importance du dialogue, Bruxelles, Conférence sur le dialogue interculturel, 20 mars 2002, Speech 02/114, disponible en ligne :

http://europa.eu.int/rapid/pressReleasesAction.do?reference=SPEECH/02/114\&format= DOC\&aged $=1$ \&language $=\mathrm{FR} \&$ guiLanguage $=\mathrm{f} ;$ Prodi $\mathrm{R}$., $L$ 'UE, le dialogue avec les religions et la paix, Camaldoli, 14 juillet 2002, « Rencontre christianisme et démocratie dans l'avenir de l'Europe », disponible en ligne :

http://europa.eu.int/rapid/pressReleases Action.do?reference=SPEECH/02/345\&format= DOC\&aged $=1$ \&language $=$ FR\&guiLanguage $=$ en

14 . Prodi R., L'Europe élargie - Une politique de proximité comme clé de la stabilité, Bruxelles, 5 décembre 2002, Speech 02/619, disponible en ligne :

http://europa.eu.int/rapid/pressReleasesAction.do?reference=SPEECH/02/619\&format= PDF\&aged $=1$ \&language $=F R \&$ guiLanguage $=$ en

15. Ibid, p. 3 .

16. Ibid, p. 4. 
qu'une adhésion », et qui viserait à terme de «tout» mettre en commun avec les voisins, "sauf les institutions " ${ }^{17}$. L'initiative proposée s'inscrit ainsi entre la pratique européenne de l'élargissement et celle du partenariat. Les mesures relevant de considérations de sécurité ne sont pas absentes de cette perspective, mais elles constituent un domaine d'action parmi d'autres.

Les orientations suggérées par le discours ECSA de Romano Prodi seront très largement reprises par la communication Europe élargie - Voisinage, tant au niveau du cadre géographique du voisinage (inclusion des pays du processus euro-méditerranéen) ${ }^{18}$ qu'au niveau des contenus. La communication articule la logique fondamentale de la PEV dans les termes suivants :

«L'Union devrait offrir à ses voisins de nouvelles perspectives d'intégration économique en contrepartie de leurs progrès concrets dans le respect des valeurs communes et la mise en œuvre effective des réformes politiques, économiques et institutionnelles, notamment dans l'alignement de leur législation sur l'acquis. A cette fin, l'Union devrait offrir [...] une perspective de participation au marché intérieur ainsi que la poursuite de l'intégration et de la libéralisation afin de promouvoir la libre circulation des personnes, des biens, des services et des capitaux (les quatre libertés) 19 ".

La question du voisinage s'inscrit clairement ici dans une reformulation des liens entre intégration et élargissement. Il s'agit moins de suivre la pratique de l'intégration par l'élargissement, que d'

« œuvrer avec [les] partenaires pour réduire la pauvreté et créer un espace de prospérité et de valeurs partagées, fondé sur une intégration économique accrue, des relations politiques et culturelles plus intenses, une coopération transfrontalière renforcée et une prévention conjointe des conflits... ${ }^{20}$ "

A la pratique de l'élargissement de l'UE, qui domine depuis plus d'une décennie les débats au sein des arènes européennes sur les relations avec l'étranger proche de l'Union, se substitue ici l'esquisse d'une pratique alternative - la constitution d'un «espace » aux contenus et aux dynamiques très vagues - dont toute l'ambiguïté est de mobiliser des éléments liés à l'élargissement sans pour autant les associer à une perspective d'adhésion : soutien aux

17. Ibid, p. 4.

18. Conseil européen, Conseil européen de Copenhague, Conclusions de la présidence, Bruxelles, 29 janvier 2003, 15917/02, POLGEN 84, p. 6, point 22, disponible en ligne :

http://www.consilium.europa.eu/ueDocs/cms_Data/docs/pressData/en/ec/73842.pdf

19. Commission européenne, L'Europe élargie - Voisinage, op. cit., p. 4. Soulignement d'origine. 20. Ibid, p. 9. Soulignement d'origine. 
réformes économiques, politiques et sociales, passant notamment par un alignement réglementaire et législatif sur les pratiques européennes, et promotion des «quatre libertés », entre autres.

Cet élément des "quatre libertés », de par l'insistance dont il fait l'objet, à la fois dans le discours ECSA de Romano Prodi et dans la communication Europe élargie - Voisinage, mérite davantage d'attention ${ }^{21}$. Le thème de la libre circulation des personnes, tout particulièrement, constitue une ligne de réflexion majeure, en ce qu'il s'oppose à première vue à une lecture sécuritaire de l'immigration comme menace, exprimée en termes de contrôle des frontières et des flux migratoires. La communication Europe élargie Voisinage dispose d'une section entière dévolue à cette question ${ }^{22}$, qui stipule en particulier que :

« dans un contexte de déclin démographique et de vieillissement de la population, de mondialisation et de spécialisation, il peut être avantageux, pour l'Union et ses voisins, d'instaurer des mécanismes permettant aux travailleurs de passer d'un territoire à l'autre pour se rendre là où leurs compétences sont le plus nécessaires - même si l'objectif à long terme reste la libre circulation des personnes et des travailleurs ${ }^{23}$ ».

Comme le note Elspeth Guild 24, ce constat s'accompagne cependant d'un ensemble de propositions contrastées, dont deux paraissent en contradiction ouverte avec l'objectif initial, à savoir le soutien aux «efforts déployés par les pays voisins pour lutter contre la migration clandestine » qui passe par l'instauration de "mécanismes efficaces en cas de retour [...], plus spécialement pour les migrants en transit", et "la conclusion d'accords de réadmission avec tous les pays voisins » comme "jalon dans la lutte commune contre la migration clandestine » 25 . L'impression générale donnée par le document est, à ce titre, celle d'une difficulté à jouer d'une position alternative dans un contexte politique européen surdéterminé par la mise en place d'un agenda en matière de sécurité qui fait du contrôle des frontières et des migrations un thème majeur.

Cette impression est confirmée dans les étapes ultérieures de l'élaboration de la PEV. Un document qu'il convient de mentionner dans cette perspective

21. Voir sur cette question : Guild E., «What is a neighbour? Examining the EU Neighbourhood Policy from the perspective of movement of persons ", Yalta, papier présenté au Forum "Western NIS for Refugee Assisting NGOs», 1-3 juin 2005, disponible en ligne: http://www.ecre.org/research/What\%20is\%20a\%20NeighbourEGuild.pdf

22 . Sous l'intitulé : «Perspectives en matière de migration légale et de circulation des personnes ", Commission européenne, L’Europe élargie - Voisinage, op. cit., pp. 11-12.

23. Ibid, p. 11.

24. Guild E., "What is a neighbour?", op. cit.

25. Commission européenne, L'Europe élargie - Voisinage, op. cit., p. 12. 
est le texte portant sur la "Stratégie européenne de sécurité » (SES), développé par Javier Solana, en sa qualité de Haut Représentant pour la PESC (Politique étrangère de sécurité commune), et son équipe ${ }^{26}$. La lecture des évolutions de l'environnement international dont participe la SES se structure autour du constat de l'existence de «nouvelles menaces 27 ». Elle s'inscrit donc à ce titre dans le discours de l'«insécurité globale » qui prospère au sein des arènes internationales depuis la fin de la Guerre froide ${ }^{28}$. Sous l'intitulé "Construire la sécurité dans notre voisinage 29 ", la SES reformule également la PEV, en notant qu'il est

« dans l'intérêt de l'Europe que les pays situés à ses frontières soient bien gouvernés. Les voisins engagés dans des conflits violents, les Etats faibles où la criminalité organisée se répand, les sociétés défaillantes ou une croissance démographique explosive aux frontières de l'Europe constituent pour elle autant de problèmes 30 ».

Il s'agit alors de "promouvoir, à l'Est de l'UE et aux frontières du bassin méditerranéen, un ensemble de pays bien gouvernés » avec lesquels pourraient être envisagées «des relations étroites, fondées sur la coopération » ${ }^{31}$. Ce mouvement n'est pas seulement rhétorique, et entraîne des effets certains en matière de formulation des politiques à mener et de leurs enjeux. Ces effets transparaissent ainsi nettement dans la deuxième communication majeure de la Commission sur la PEV, le document d'orientation de mai 2004 32, qui précède de quelques mois les propositions pour les premiers plans d'action bilatéraux visant à mettre en œuvre la politique.

Les changements apportés par le document d'orientation de mai 2004 jouent en fait à deux niveaux. Ils entérinent tout d'abord la réorientation générale de la PEV développée dans la SES, et notamment la mise sous coupe

26. Solana J., Une Europe sûre dans un monde meilleur. Stratégie européenne de sécurité Document adopté par les chefs d'Etat et de gouvernement, réunis en Conseil européen à Bruxelles, le 12 décembre 2003, disponible en ligne:

http://www.iss-eu.org/solana/solanaf.pdf

27 . «Une agression de grande envergure contre un des Etats-membres est actuellement improbable. En revanche, l'Europe est confrontée à de nouvelles menaces, qui sont plus variées, moins visibles et moins prévisibles ", ibid., p. 7.

28. Voir Bigo D., "Grands débats dans un petit monde. Les débats en relations internationales et leur lien avec le monde de la sécurité », Cultures E Conflits, $\mathrm{n}^{\circ} 19-20,1995$, pp. 7-48. La SES adopte position pour une lecture maximisante de ces soi-disant nouvelles menaces, soulignant notamment les risques d'une conjonction entre « un terrorisme fermement résolu à user d'une violence maximale, l'accès à des armes de destruction massive, la criminalité organisée, l'affaiblissement du système étatique et la privatisation de la force »(SES, op. cit., p. 11).

29. Ibid., pp. 15-17.

30 . Ibid., p. 15.

31. Ibid.

32. Commission européenne, Politique européenne de voisinage. Document d'orientation. Bruxelles, COM (2004) 373 Final, 12 mai 2004, disponible en ligne : http ://europa.eu.int/comm/world/enp/pdf/strategy/Strategy_Paper_FR.pdf 
réglée des logiques de soutien aux dynamiques de réforme dans les pays du voisinage à des considérations de sécurité. La SES, dans ce cadre, devient une référence centrale ${ }^{33}$. Ce développement a un deuxième effet, à savoir une mise en avant significative des dispositions relevant des questions de sécurité, et notamment les thématiques du contrôle des frontières et de la gestion des flux migratoires. La dimension Justice et Affaires intérieures (JAI) devient, à ce titre, le premier domaine de compétence communautaire spécifiquement mentionné dans la section « Principes et portée » du document ${ }^{34}$.

Ce mouvement s'illustre notamment par la disparition de toute mention des «quatre libertés 35 » et le transfert des points traitant de la circulation des personnes et des migrations dans la section JAI du document. Si cette dernière s'ouvre par l'affirmation que "la PEV vise à éviter tout nouvean clivage aux frontières de l'Europe élargie », et le constat de l'existence d'un «intérêt commun » à divers niveaux entre l'UE et les pays du voisinage ${ }^{36}$, elle réitère néanmoins l'idée que «la gestion des frontières sera très probablement un thème prioritaire ", notamment dans le but de "faciliter les déplacements légitimes » ${ }^{37}$. L'intérêt commun évoqué est par ailleurs largement défini sous l'angle de la prise en compte des «nouvelles menaces» évoquées dans la SES : "Les partenaires sont également confrontés à des défis accrus dans le domaine de la justice et des affaires intérieures, tels que la pression migratoire en provenance des pays tiers, la traite des êtres bumains et le terrorisme 38 ». La seule suggestion selon laquelle une libéralisation de la circulation des personnes pourrait être envisagée tient dans les deux phrases suivantes : «L'UE pourrait également examiner la possibilité d'assouplir les règles d'octroi des visas. Cet assouplissement concédé par une partie devra être toutefois compensé par des actions efficaces de l'autre 39 ». On passe donc d'une offre de régime de libre circulation à la mise en place d'un système de contrôle différencié, visant à faciliter la circulation de certaines catégories de personnes dites « légitimes » tout en augmentant les capacités à contrôler les mouvements considérés comme « illégitimes » 40 .

33. "L'importance d'une politique de voisinage est également mise en évidence dans la stratégie européenne de sécurité [...] qui déclare qu'il appartient à l'UE d'apporter une contribution à la stabilité et à la bonne gouvernance dans notre voisinage immédiat et à promouvoir un cercle de pays bien gouvernés à l'est de l'Union européenne et sur le pourtour méditerranéen ». Commission européenne, Document d'orientation, op. cit., p. 6.

34 . "La PEV [...] peut également aider l'Union à atteindre ses objectifs dans le domaine de la justice et des affaires intérieures, notamment pour ce qui est de la lutte contre le crime organisé et la corruption, le blanchiment de capitaux et toutes les formes de trafic, ainsi qu'en ce qui concerne les questions liées à la migration ", ibid.

35. Le langage de substitution employé dans le document d'orientation est en effet beaucoup plus vague sur les liens entre voisinage et marché intérieur. Il s'agit par exemple de "laisser entrevoir aux pays voisins une possible participation au marché intérieur de l'UE », Commission européenne, Politique de voisinage. Document d'orientation, op. cit., p. 14.

36. Ibid., p. 18.

37 . Ibid.

38. Ibid.

39. Ibid.

40. Ce qui illustre le respect de l'«impératif de mobilité» que Didier Bigo identifie comme l'une 
On en revient donc à une formulation proche de celle exprimée dans la lettre britannique de janvier 2002 sur la relance des relations entre l'UE et ses contiguités géographiques, et à l'établissement, au cœur de la PEV, d'une narration ${ }^{41}$ inscrivant le voisinage comme un espace où se développent des insécurités potentielles, plus ou moins interconnectées, qu'il convient de contrer par le biais d'une politique promouvant des pratiques de «bonne gouvernance » dans une optique sécuritaire. Il est important d'insister sur ce point : nous ne sommes pas ici en présence d'un renforcement de la «dimension de sécurité » de la PEV comme l'analysent certains commentateurs ${ }^{42}$, mais plutôt d'une restructuration générale des orientations de la PEV par une « grammaire» ou une «formation de sécurité » ${ }^{3}$. Cette restructuration ne s'opère pas de manière linéaire et «naturelle ", mais bien au détriment de prises de position qui constituaient la PEV comme un espace propice au développement d'une perspective alternative à une lecture sécuritaire. Cette restructuration, par ailleurs, est paradoxale : elle intervient dans un espace où la plupart des discours autorisés sont émis depuis des positions situées dans les secteurs politique, diplomatique ou des relations extérieures communautaires, positions qui semblent ne pas permettre aux agents qui les occupent de produire un discours autorisé sur la « menace ». Pour mieux comprendre comment ce mouvement opère, il convient donc d'analyser plus avant les interactions entre les pratiques de labellisation analysées ici et celles des agents sociaux qui se sont investis, au jour le jour, dans l'élaboration de la PEV.

\section{Investir le voisinage : jeux sectoriels dans la mise en place de la PEV}

Pour ce faire, nous nous penchons ici sur les usages qui sont faits des discours autorisés sur le voisinage dans les pratiques des agents chargés de l'élaboration de la PEV. Mener ce travail implique cependant une réflexion préalable sur les agents eux-mêmes et sur leur position au sein des arènes communautaires. Nous nous focalisons ici clairement sur l'analyse d'une dimension particulière de ces espaces sociaux transnationaux qui constituent l'Europe ${ }^{44}$, à savoir le secteur institutionnel communautaire, et tout particulièrement sur les arènes bureaucratiques au sein desquelles la politique de voisinage a été principalement élaborée.

Le groupe d'agents le plus visible dans la genèse et la mise en œuvre de la PEV est à localiser au sein des structures de la Commission, dans la direction générale Relations extérieures (DG Relex). La communication Europe élar-

des trois composantes du dispositif ban-optique. Bigo D., «Globalized (in)security: the field and the Ban-opticon », in Bigo D., Tsoukala A. (eds.), Illiberal practices of liberal regimes: the (in) security games, Paris, Centre d'études sur les conflits, L'Harmattan, pp. 5-49.

41. Le "récit de la menace" pour Alexandra Goujon. Goujon A., "L'Europe élargie en quête d'identité », op. cit.

42. Lynch D., "The security dimension of the European Neighbourhood Policy », op. cit.

43. Huysmans J., "Dire et écrire la sécurité : le dilemme normatif des études de sécurité ». Cultures \& Conflits, $\mathrm{n}^{\circ} 31-32,1998, \mathrm{pp}$. 177-202.

44. Cohen A., Dezalay Y., Marchetti D., «Esprits d'Etat, entrepreneurs d'Europe ", Actes de la recherche en sciences sociales, ${ }^{\circ} 166-167,2007$, pp. 5-13. 
gie - Voisinage a en effet été réalisée par un groupe réduit d'officiels de cette direction générale, appartenant aux unités chargées des relations entre l'UE et les anciennes républiques soviétiques, sous la supervision rapprochée du commissaire en charge du portefeuille des Relations extérieures à l'époque, Chris Patten 45. Ce noyau initial a ensuite été étendu, à partir de juillet 2003 et à travers la mise en place du groupe de travail «Europe élargie " ("Wider Europe task force »), à d'autres officiels de la même direction générale, mais également à un groupe important d'officiels issus de la DG Elargissement ${ }^{46}$. A ce titre, il est légitime d'affirmer que ce n'est pas «la Commission» dans son ensemble qui s'est saisie de la question de la politique de voisinage, mais plutôt un groupe relativement réduit d'officiels partageant une attache institutionnelle formelle, et un ensemble de pratiques professionnelles relativement homogène lié à la gestion des relations extérieures communautaires. Cet angle d'approche est inspiré de la réflexion menée par Yves Buchet de Neuilly sur les acteurs de la politique étrangère européenne et leurs pratiques :

«L'espace des relations internationales est marqué par une forte division du travail diplomatique. L'intégration progressive des Etats dans la configuration mondiale est le travail d'individus spécialisés dans des secteurs de l'activité publique spécifiques [...] le plus souvent issus des ministères en charge de ces politiques à l'intérieur des Etats. Ce sont des "experts", par opposition aux "généralistes" que constituent les fonctionnaires des ministères des Affaires étrangères 47 ».

Buchet de Neuilly porte cette distinction entre «professionnels de la gestion des relations économiques extérieures» et "professionnels de la diplomatie politique» dans une analyse des arènes européennes et des interactions complexes entre ces deux secteurs spécifiques, en soulignant notamment que ce clivage n'épouse pas la topologie des institutions bruxelloises, mais constitue une ligne de démarcation transversale, qui se retrouve au sein de la Commission elle-même.

45. Plus spécifiquement, ces officiels appartenaient à l'unité E/1 («Questions horizontales ») de la direction E («Europe de l'Est, Caucase, Républiques d'Asie centrale ») au sein de la DGA2 («Europe et Asie centrale, Moyen-Orient, Méditerranée du sud »). Entretien, DG Relex, juin 2005.

46. Parmi lesquels deux chefs d'unités (Rutger Wissels, Andreas Herdina) et Michael Leigh, l'ancien chef de la direction C ( Bulgarie, Chypre, Malte, Roumanie, Turquie »), nommé au début de 2003 directeur de la DGA-2 à DG Relex, et qui prend la direction du groupe de travail «Europe élargie ». Il est intéressant de noter par ailleurs qu'en 2004, l'ancien directeur général de la DG Elargissement, Eneko Landaburu Illaremendi, est nommé à la tête de DG Relex. Michael Leigh, par ailleurs, retourne en 2005 prendre la tête de la DG Elargissement. Andreas Herdina prendra en janvier 2005 la tête de l'unité D/2 ( Coordination sectorielle de la PEV») au sein de la DG Relex, tandis que Rutger Wissels sera nommé directeur de la direction D ("Coordination de la PEV »). Il s'agit là d'une dynamique de circulation particulièrement intéressante, dans la mesure où elle démontre finalement la proximité, en termes de pratiques professionnelles, entre les deux directions générales.

47. Buchet de Neuilly Y., L'Europe de la politique étrangère, Paris, Economica, 2005, p. 101. 
Les pratiques relatives à la gestion des relations économiques extérieures, dans cette lecture, se distinguent des pratiques de la diplomatie politique en ce qu'elles n'impliquent pas tant une intervention de nature ponctuelle

«au nom d'un Etat, d'une organisation internationale ou d'une organisation non gouvernementale, pour prendre position sur les évolutions politiques internes et internationales d'Etats tiers, pour réagir à certaines confrontations physiques à l'intérieur ou entre des Etats et $[\ldots]$ pour modifier ces situations 48 ».

qu'une activité inscrite à la fois dans la quotidienneté et dans une temporalité plus longue que celle de la diplomatie politique ${ }^{49}$, faisant appel à des savoirs et savoir-faire distincts.

Ces pratiques de gestion des relations extérieures constituent l'élément commun aux officiels rassemblés dans le groupe de travail «Europe élargie». Qu'ils proviennent de la DG Relex ou de la DG Elargissement, ils partagent en effet certaines activités professionnelles, incluant : des tâches de coordination entre les services dits extérieurs de la Commission, mais aussi entre les délégations sur le terrain et le siège à Bruxelles; la conduite des contacts, formels et informels, avec les officiels gouvernementaux des pays concernés (par le biais de missions sur le terrain ou de réunions à Bruxelles); et une activité d'évaluation des politiques communautaires, de proposition (par le biais de textes législatifs ou de communications) ainsi que de programmation de l'assistance financière communautaire (la gestion quotidienne étant, elle, dévolue à l'office de coopération EuropeAid). La distinction entre officiels issus de la DG Relex et officiels issus de la DG Elargissement renvoie ici plus à l'expérience des modes de fonctionnement spécifique de cette dernière qu'à une différence fondamentale entre la pratique de l'élargissement et celle de la gestion des relations extérieures communautaires. La DG Elargissement est organisée, à l'image de la DG Relex, en directions et unités géographiques, chaque unité étant responsable du suivi d'un pays spécifique. Cependant, en plus de leurs activités " géographiques », les officiels de la DG Elargissement sont responsables du suivi d'un «secteur », c'est-à-dire d'un domaine technique spécifique, transversal aux relations avec tous les pays candidats (par exemple le nucléaire civil, les transports, les infrastructures routières, etc.). C'est à ce titre, notamment, qu'un de ces officiels issus de la DG Elargissement, contacté dans le cadre de notre recherche, situe ses activités et celles de ses collègues dans "l'expérience des processus de transformation qui vient de l'accompagnement

48. Ibid., p. 2.

49. Nous abandonnons cependant la dénomination de relations «économiques " extérieures, pour rendre mieux compte de la gamme de pratiques qui structurent les activités de ces professionnels, notamment en matière d'assistance technique, qui sont plus larges que le maniement des instruments économiques de l'action internationale. 
de l'élargissement [...], l'expérience sectorielle dans les relations extérieures 50 ». L'identification professionnelle des deux groupes à la gestion des relations extérieures demeure donc forte. Le profil qui se dégage de ces éléments s'organise donc autour de la reconnaissance, de la revendication et de la valorisation d'un savoir et d'un savoir-faire spécifiques impliquant une familiarité pratique avec un pays ou une zone géographique particulière, les mécanismes de l'assistance financière et technique communautaire, ou encore la routine des interactions avec les officiels gouvernementaux des pays tiers, dans une perspective temporelle plus longue que celle de la diplomatie politique.

L'implication de ce groupe spécifique de professionnels de la gestion des relations extérieures communautaires dans l'élaboration de la PEV participe de plusieurs dynamiques différentes. Il y a, tout d'abord, la correspondance entre le discours dominant qui émerge fin 2002 sur les relations entre l'UE et ses voisins, et les pratiques qui caractérisent leurs activités. Si l'on reprend les orientations développées dans l'allocution de Romano Prodi devant le congrès ECSA, on note que cette dernière constitue une prise de position en faveur des pratiques des relations extérieures communautaires, dans le contexte des travaux de la Convention pour l'avenir de l'Europe :

« La Commission vient juste de présenter sa deuxième communication à la Convention [...]. Nous avons [...] plaidé en faveur d'une Commission forte, ce qui, la Commission étant la gardienne de l'intérêt communautaire, renforcera l'Union [...]. La méthode communautaire sera très utile dans le domaine des relations extérieures, nous permettant de jouer un rôle d'intermédiaire pour négocier des arrangements particuliers et trouver des solutions ad hoc 51 ».

Le discours souligne par ailleurs que, dans le cadre des relations avec les voisins, «c'est à la Commission qu'il incombe de trouver un moyen d'améliorer les relations avec l'ensemble de ces pays 52 ». Les propositions développées par Prodi aménagent donc, de fait, un espace privilégié pour les tenants des relations extérieures communautaires au sein de la Commission. Il semble que l'arrivée d'officiels de la DG Elargissement dans le processus ne soit pas tant le reflet d'une nécessité fonctionnelle, d'un besoin essentiel en matière d'expertise sectorielle, que le corollaire de cet aménagement, surdéterminé par d'autres facteurs tels que l'implication du commissaire à l'élargissement de l'époque, Gunter Verheugen, sur la question du voisinage ${ }^{53}$.

50. Entretien, DG Relex, novembre 2006.

51 . Prodi R., «L'Europe élargie », op. cit., p. 2.

52. Ibid., p. 4.

53. Le récit le plus fréquemment proposé dans nos entretiens est que Verheugen cherchait littéralement à «placer» les officiels sous sa responsabilité dans la perspective d'une réduction majeure des effectifs de la DG Elargissement après mai 2004. 
Le groupe de travail «Europe élargie » va donc, dans un premier temps, occuper de façon solitaire l'espace ouvert par le lancement de la politique de voisinage. Les officiels y participant vont devenir les principaux acteurs du développement de la PEV tout au long de l'année 2003, non seulement au sein de la Commission, mais plus largement dans les arènes institutionnelles bruxelloises. Cette position s'explique en partie par le fait que la constitution d'une structure spécifique dédiée à la politique de voisinage n'est reflétée dans aucune autre direction générale de la Commission, pas plus que dans les services du secrétariat général du Conseil, pourtant supposé être l'interlocuteur privilégié dans les matières afférentes au deuxième pilier ${ }^{54}$. La coordination entre les services de la Commission est restée largement lettre morte, malgré la création d'une structure interservices formelle par le secrétariat général de la Commission en juillet 2003. Bien sûr, il ne faut pas négliger l'importance des contacts informels, mais le groupe de travail « Europe élargie » a largement fonctionné en vase clos, n'entretenant de contacts poussés qu'avec les officiels des unités géographiques de la DG Relex ${ }^{55}$. Pour ce qui concerne le Secrétariat général du Conseil, et du propre aveu des officiels impliqués à l'époque, les contacts avec la Commission se sont déroulés sur un mode largement routinier, sans impulsion significative de la part des Etats-membres ou de l'équipe du Haut Représentant ${ }^{56}$. Enfin, les contacts avec le Parlement européen sont, à ce moment précis, épisodiques au mieux ${ }^{57}$. Ce sont donc les officiels du groupe de travail qui vont entreprendre de contacter les services gouvernementaux des pays concernés par l'initiative, établir les premiers rapports de pays préliminaires à l'établissement des plans d'action bilatéraux par laquelle la PEV est actuellement mise en ouvre, et se charger de rédiger le document d'orientation de mai 2004.

C'est sur ce dernier point, cependant, qu'une question se pose. Comme nous l'avons vu, le document d'orientation entérine un changement majeur dans l'optique de la PEV, en la reformulant selon une orientation sécuritaire. Comment comprendre cette évolution, alors que les principaux agents impli-

54. Au sein du secrétariat général du Conseil, l'élaboration de la PEV a été suivie à l'époque par deux unités positionnées dans deux directions différentes et dépendant de la DG E («Relations économiques extérieures, affaires politico-militaires») - l'unité 5A au sein de la direction 5 ( "Méditerranée, Moyen-Orient, Afrique, Asie ») et l’unité 6A au sein de la direction 6 («Balkans occidentaux, Europe de l'Est, Asie centrale »). La PEV a également été suivie par trois groupes de travail différents au sein du Conseil, le groupe $\mathrm{Ma} / \mathrm{Ma}$ (« Maghreb et Mashreq»), MoyenOrient/Golfe, et COEST ( Asie centrale et Europe de l'Est ») auxquels il arrive parfois d'organiser des réunions communes. Entretien, DG E, Secrétariat général du Conseil, avril 2005.

55 . Entretien, DG Relex, Commission européenne, avril 2005.

56. Entretien, DG E, Secrétariat général du Conseil, avril 2005.

57. Le Parlement européen n'est que très marginalement impliqué à ce stade. Il a émis en novembre 2003 une opinion sur la communication Europe élargie - Voisinage, dont le seul impact notable a été la confirmation de l'incorporation des trois républiques du Caucase méridional (Arménie, Azerbaïdjan, Géorgie), orientation qui avait cependant déjà été amorcée auparavant (entretien, DG Relex, juin 2005). La position du Parlement a cependant été renforcée par la suite, mais surtout du fait de son implication dans le jeu budgétaire et les négociations autour des instruments de financement des relations extérieures, dont l'instrument de voisinage (entretien, DG Relex, novembre 2006). 
qués dans l'élaboration de la politique restent apparemment les mêmes ? Il semble que des éléments soient à rechercher à la fois dans des rivalités au sein des arènes européennes, mais aussi dans des développements propres à la Commission européenne, à partir de la fin 2003. Ces rivalités, dans tous les cas, signalent un investissement de la part d'autres agents dans la configuration ouverte par la PEV. Cet investissement provient, d'abord, du secteur communautaire des professionnels de la diplomatie politique : d'une part le Haut Représentant pour la PESC, d'autre part les représentants des Etatsmembres dans le cadre du deuxième pilier. L'adoption de la SES par le Conseil est un premier marqueur significatif de cet investissement.

Deuxième marqueur important, les tensions au printemps 2004 entre la Commission et le Conseil 58 sur l'organisation des rapports entre les deux institutions dans le cadre de la PEV. Ces tensions ont porté, plus spécifiquement, sur le stade d'avancement dans les contacts entre les officiels chargés de l'élaboration de la PEV et les autorités gouvernementales des pays du voisinage. Ces contacts devaient officiellement permettre au groupe de travail « Europe élargie », en coopération avec les unités géographiques concernées, de rédiger des rapports par pays pour proposer une évaluation préliminaire à la négociation des premiers plans d'action. Ils ont cependant été présentés, par le COREPER (Comité des représentants permanents) et les officiels chargés du suivi de la PEV au sein du secrétariat général du Conseil, comme une amorce de négociations, procédure pour laquelle la Commission devait recevoir un mandat de la part du Conseil 59. L'argument du mandat a été mis en avant pour faire paraître devant le COREPER, à plusieurs reprises au cours des mois de février et mars 2004, les officiels chargés du dossier, et a eu pour corollaire une formalisation significative des rapports entre Commission et Conseil, notamment au travers de l'établissement d'interlocuteurs spécifiques à tous les niveaux entre les deux institutions. Et de fait, la lecture dominante dans nos entretiens au secrétariat général $\mathrm{du}$ Conseil a été que ces tensions constituaient un «ajustement institutionnel» dans une situation où la Commission était perçue comme ne donnant pas suffisamment d'informations sur ses activités dans le cadre de la PEV 60. Au-delà de la PEV, cependant, il est clair que ces rivalités s'inscrivent dans la dynamique plus large de la délimitation du contrôle exercé sur les secteurs des relations

58. Nous ne détaillons pas ici les divergences qui ont vu le jour entre les représentants des différents Etats-membres au sein du Conseil sur la politique de voisinage, notamment du fait de la grande discrétion qu'ont entretenue nos interlocuteurs à ce sujet. De telles divergences existent cependant. Elles portent en particulier sur le principe de répartition de l'assistance financière et technique communautaire entre les deux grands «espaces» géographiques couverts par la PEV (Méditerranée et espace post-soviétique), les représentants des Etats riverains de la Méditerranée souhaitant en particulier que les pays du Maghreb (le Maroc au premier chef) ne soient pas désavantagés par rapport aux pays de l'espace post-soviétique (principalement l'Ukraine), ces derniers constituant en revanche une priorité pour les gouvernements allemand et polonais notamment.

59. Entretien, DG Relex, avril 2005. Voir également Goujon A., « L’Europe élargie en quête de d'identité », op. cit., p. 149.

60. Entretien, DG E, Secrétariat général du Conseil, avril 2005. 
extérieures et de la diplomatie communautaires, entre professionnels de la diplomatie politique et professionnels de la gestion des relations extérieures.

Il convient néanmoins de souligner une deuxième dimension de ces tensions, impliquant les questions de sécurité. La PEV, comme nous l'avons dit, touche à une série de questions politiquement sensibles dans les arènes politiques nationales et européennes : gestion des frontières, contrôle des flux migratoires, ou coopération avec les pays tiers en matière de sécurité. Si l'on peut conclure que, dans le cas des rivalités entre Conseil et Commission au début de 2004, "le Conseil s'impose comme le décideur ultime des orientations politiques 61 ", il ne faut pas simplement limiter cette (re)prise de contrôle à la dimension des relations extérieures. Ainsi, au sein de la Commission comme au sein du Secrétariat général du Conseil, persiste cette idée que les gouvernements des Etats-membres prennent surtout intérêt à la PEV en ce qu'elle implique ce que l'un de nos interlocuteurs baptise «les questions du côté obscur 62 », à savoir les thématiques de sécurité. Un officiel, interrogé sur cette question, la résume ainsi : "Dans la présentation de nos intérêts dans la politique de voisinage, vous avez raison. Dans le fond de nos relations avec les voisins, ça n'est pas le cas [...], c'est lié à cette atmosphère générale un peu défensive de l'Union européenne ", tout en se sentant obligé de rajouter :

"Sur le fond, c'est légitime [...], on a des menaces concrètes [...]. On sait que s'il y a des défaillances dans l'Etat de droit dans les pays voisins, tout pète [...], c'est l'intérêt partagé pas seulement par ceux qui sont intéressés à la sécurité policière [...], c’est légitime parce que ça a un profil aigu 63 ».

Il y a donc non seulement reformulation de la PEV par l'intégration d'une « grammaire » de la sécurité, mais également socialisation à une perspective sécuritaire au sein même de secteurs qui ne touchent pourtant que de façon très lointaine à ces thématiques, et des effets de contrainte majeurs sur le travail des officiels chargés de la conduite de la PEV 64.

Cette reformulation participe par ailleurs de dynamiques travaillant au sein même de la Commission. Il est important, pour bien amener ce point, de rappeler au préalable que la Commission est loin d'être une arène pacifiée,

61. Goujon A., «L'Europe élargie en quête d'identité », op. cit., p. 149.

62 . «Dark side issues », Entretien, DG Relex, avril 2005 ; Entretien, DG E, Secrétariat général du Conseil, avril 2005.

63. Entretien, DG Relex, novembre 2006.

64. Un autre officiel interrogé à l'époque sur son travail au sein de la mission d'assistance aux frontières en Moldavie et en Ukraine (EUBAM), et qui s'occupait plus particulièrement des questions douanières, soulignait : «Bon, je parle pas de circulation de personnes, c'est pas mon sujet et c'est pas normal que j'en parle. Ça, c'est un problème, et on sait très bien quelles sont les sensibilités des Etats-membres. La Commission peut faire des propositions, il y a des aspects sur lesquels, nous, on a compétence, mais pas sur ceux-là », (Entretien, DG Relex, novembre 2006). 
exempte de rivalités bureaucratiques entre services. Des frictions sont ainsi apparues autour de la PEV pour ce qui concerne le rôle des officiels de la DG Relex. Pour ceux-ci, il semblait naturel d'assumer une fonction de coordination, impliquant la centralisation des initiatives provenant d'autres services. Cette position, basée sur la revendication d'un savoir légitime en matière de relations extérieures, de contacts avec les voisins et d'une compétence généraliste propice à une activité de coordination, se caractérise notamment par l'adoption de tout un vocabulaire, selon lequel la DG Relex est la «lead DG " ou «chef de file », les autres directions générales étant considérées comme des «line DG»65. Cependant, dans le cadre de la PEV comme dans d'autres domaines des relations extérieures communautaires, cette distinction fait l'objet d'un rejet de la part des intéressés, soit sur le mode argumentaire, soit sur le mode de l'ironie, mais toujours en soulignant la spécificité du point de vue de la DG Relex, et en contestant ce rôle de coordination : "Je ne sais pas ce que les Relex appellent "line DG", je veux dire, ils appellent tout le monde "line DG", mais selon moi, nous nous voyons plutôt comme des partenaires égaux 66 ».

La réflexion de ce dernier officiel, en poste à la DG justice, liberté et sécurité (JLS), est particulièrement intéressante dans le cadre des relations entre la DG Relex et la DG JLS sur les questions de voisinage. Les points de vue collectés au cours de nos entretiens sont assez éclairants quant à la position spécifique occupée par la seconde dans les arènes communautaires. Ainsi, la DG JLS est à la fois identifiée comme "parlant aux ministères de l'Intérieur des Etats-membres », mais également comme étant contrainte par les autorités nationales compétentes dans ses domaines d'action, au point de vouloir «faire en externe ce qu'elle ne peut pas faire en interne ", c'est-à-dire d'inscrire ses activités dans le cadre des relations extérieures communautaires où la répartition formelle des compétences est plus favorable aux agents communautaires ${ }^{67}$. Cette inscription génère logiquement des frictions avec les agents détenteurs des positions les plus pérennes dans le secteur de la gestion des relations extérieures communautaires, d'autant plus que les services de la DG JLS sont des entrants récents 68 .

Dans le cadre de la PEV, ces rivalités sont plus spécifiquement cristallisées sur les questions relatives à la libre circulation des personnes, et notamment au lien entre facilitation des visas (c'est-à-dire assouplissement des règles d'obtention de visas pour certains pays tiers, ici les voisins) et conclusion d'accords de réadmission (qui autorisent la reconduite sur le territoire de chacun des signataires de migrants en situation irrégulière, qu'ils soient originaires de ce terri-

65. L'expression ne semble pas avoir d'équivalent en français, nos interlocuteurs francophones utilisant indifféremment les termes anglais.

66. Entretien, DG JLS, novembre 2006. Dans un autre entretien, un officiel, de la DG Commerce cette fois, s'est ainsi senti obligé, confronté à cette question, de développer le fait que sa propre direction était tout aussi généraliste que la DG Relex (Entretien, DG Commerce, juin 2006).

67. Entretien, DG Relex, juin 2005.

68. La DG JAI a été créée en 1999 avec l'arrivée aux affaires de la Commission Prodi. 
toire ou qu'ils y aient simplement transité). La position adoptée par la DG JLS était que la question de la réadmission constituait un aspect central de l'engagement des voisins vis-à-vis de l'UE, et une priorité de tout premier ordre ${ }^{69}$. Au sein de la DG Relex, si la question de la réadmission n'était pas minimisée, la compréhension générale était qu'il devait y avoir une forme de réciprocité entre facilitation et réadmission. Comme le souligne un de nos interlocuteurs :

«On peut effectivement dire qu'il y avait un certain degré de tension sur ces questions. Oui, JLS est en contact avec les ministères de l'Intérieur, qui sont, comment dire, conservateurs sur la question des facilitations de visas, alors que les ministères des Affaires étrangères ont tendance à être plus ouverts sur ces questions. Dans ce sens, on retrouve au niveau de la Commission ce que vous trouveriez entre ces deux ministères au niveau des Etats-membres 70 ».

Ces tensions, néanmoins, se sont résolues au bénéfice de la DG JLS, dans la mesure où la réadmission a été inscrite comme une composante majeure des relations entre l'UE et ses voisins (voir par exemple la récente conclusion d'un tel accord avec l'Ukraine), et que la dimension JLS de la plupart des accords soutenant la PEV a été renforcée par le biais des plans d'action ${ }^{71}$.

\section{Le voisinage comme label}

Cela nous amène à récapituler notre propos, pour éclairer davantage la restructuration de la PEV autour de thématiques sécuritaires. Ce mouvement est le produit, à notre sens, d'un processus complexe impliquant des rivalités, à différents niveaux, entre les agents positionnés dans les arènes institutionnelles communautaires. Ces rivalités se jouent notamment entre professionnels de la diplomatie politique et professionnels de la gestion des relations extérieures, et portent sur le contrôle des secteurs communautaires de leurs champs respectifs. Elles passent par la mobilisation et l'importation tactiques de discours et de luttes d'autres secteurs, notamment pour ce qui concerne les questions de sécurité. Les conditions de production de la PEV sont donc, au fond, largement indépendantes de la situation concrète des populations et des pays dits « voisins », qui n'est prise en compte qu'à l'aune de la structure des luttes et des rivalités qui structurent ces mêmes arènes. Loin donc de recouvrir une existence objective, le «voisinage » de l'UE est avant tout un label sous lequel sont réinscrites les pratiques analysées dans cet article, et les luttes entre agents sociaux de l'espace institutionnel communautaire qui les accompagnent.

69. Entretien, DG JLS, juin 2005.

70. Entretien, DG Relex, novembre 2006. L'officiel appartenait auparavant à la DG JLS.

71. Entretien, DG JLS, juillet 2006. 
Ces luttes prennent place dans une configuration relativement fluide des arènes communautaires, liée à une conjugaison de deux éléments : la perspective d'un traité constitutionnel porteur de changements significatifs (en particulier la création d'un poste de ministre européen des Affaires étrangères taillé sur mesure pour Javier Solana), et la complétion formelle d'un élargissement qui a été un élément majeur de légitimation du processus d'intégration européen au cours de la décennie précédente (et dont l'usage se perpétue par la réitération ad nauseam de la notion d'Europe élargie) ainsi qu'une composante significative de la revendication d'un savoir légitime par les agents des relations extérieures communautaires. L'échec du traité, tout comme la perte de visibilité de la pratique de l'élargissement, privent une partie des agents positionnés dans les secteurs communautaires d'une ressource discursive majeure. Le voisinage prend place, à ce titre, parmi la constellation de labels (processus d'association, de coopération, de « dimensionnalisation », d'élargissement ou de stabilisation) dont la production s'est notablement accélérée après 1989 prolifération dont la source est peut-être à rechercher dans l' «éparpillement » $\mathrm{du}$ «concept politique de l'Europe», qui fait dire à certains qu'il y a aujourd'hui "autant d'Europes que de fonctions assumées par cette nébuleuse continentale sur la scène internationale $\gg 72$.

C’est dans ce contexte que le deuxième élément de l'analyse présentée cidessus rentre en jeu. Le "récit de la menace », pour reprendre la terminologie d'Alexandra Goujon, intervient ici comme une pratique de labellisation alternative d'autant plus facilement mobilisable qu'elle imprègne fortement les arènes politiques nationales, mais également transnationales ${ }^{73}$. Qu'on la conceptualise comme un «effet de champ» ou comme un "piège » de la sécurité ${ }^{74}$, le fait est que cette pratique fait sens, parce qu'elle repose en partie sur un corpus discursif et pratique constitué, y compris dans les arènes européennes, et parce qu'elle fournit du sens. Elle a donc la force de l'évidence : "c'est légitime [...] parce que ça a un profil aigu ", selon la formulation de l'un de nos interlocuteurs ${ }^{75}$. La question qui se pose alors, et qui dépasse le cadre de cet article, est celle des implications politiques d'un recentrage du « concept politique d'Europe » autour d'un discours d'(in)sécurisation.

72. Dal Lago A., Mezzadra S., «Les frontières impensées de l’Europe », Lignes, n 13,2004 , pp. 67-80.

73. Voir Bigo D., « La mondialisation de l'(in)sécurité ? Réflexions sur le champ des professionnels de la gestion des inquiétudes et analytique de la transnationalisation des processus $\mathrm{d}^{\prime}$ (in)sécurisation », Cultures E Conflits, n58, 2005, pp. 53-100.

74 . Pour la première formulation, $i b i d$. Pour la seconde voir : c.a.s.e. collective, «Critical approaches to security in Europe: a networked manifesto », Security Dialogue, vol. 37, n4, pp. 443-487.

75 . Voir note 63. 